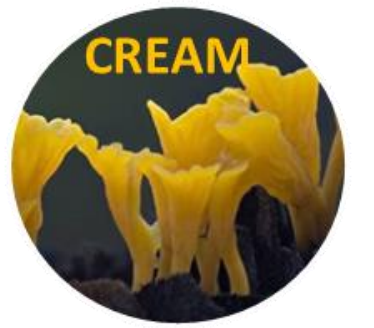

\title{
A method to stimulate production of extracellular pigments from wood- degrading fungi using a water carrier
}

\author{
Weber GL ${ }^{1}$, Boonloed $\mathrm{A}^{2}$, Naas $\mathrm{KM}^{2}$, Koesdjojo $\mathrm{MT}^{2}$, Remcho $\mathrm{VT}^{2}$ and Robinson \\ $\mathrm{SC}^{3 *}$
}

${ }^{1}$ Department of Botany and Plant Pathology, Oregon State University, 2082 Cordley Hall, Corvallis, Oregon 97331

${ }^{2}$ Department of Chemistry, Oregon State University, 296 Linus Pauling Science Center, Corvallis, Oregon 97331

${ }^{3}$ Department of Wood Science and Engineering, Oregon State University, 119 Richardson Hall, Corvallis, Oregon 97331

Weber GL, Boonloed A, Naas KM, Koesdjojo MT, Remcho VT, Robinson SC - A method to stimulate production of extracellular pigments from wood-degrading fungi using a water carrier. Current Research in Environmental \& Applied Mycology 6 (3): 218-230, Doi 10.5943/cream/6/3/10

\begin{abstract}
Blue-green, red, and yellow pigments produced by the wood-degrading fungi, Chlorociboria aeruginosalaeruginascens, Scytalidium cuboideum, and Scytalidium ganodermophthorum, respectively, have significant value within the wood products industry, and were recently shown to have potential for use as textile dyes. Such secondary metabolites have the potential to augment or replace synthetic petroleum-based compounds, and to enhance sustainability within various industries. However, production methods for these pigments are currently limited to wood-based malt-agar media and extraction with dichloromethane (DCM). This method of pigment production, while effective, limits quantity, does not yet provide enough material for use in commercial-scale applications, and requires a solvent for extraction. This study looked at the established shake-culture method for growing fungi and determined parameters required for optimizing pigment production (in contrast to increasing growth rate) for the three aforementioned fungi while keeping the pigments dispersed in water. Scytalidium cuboideum reached maximum pigment production in 16 days, $S$. ganodermophthorum in 24 days, $C$. aeruginosa in 48 days, and $C$. aeruginascens in 28 days when grown on a shaker at 110 RPM. The pigments remained dispersed in the medium throughout the test and were able to be used directly in a water carrier and extracted out with DCM. This growth method should enable large-scale production of these pigments suitable for commercialization.
\end{abstract}

Keywords - Chlorociboria aeruginosa - Chlorociboria aeruginascens - fungal pigment - Scytalidium cuboideum - Scytalidium ganodermophthorum - xylindein

\section{Introduction}

In nature and in culture, fungi produce a dizzying array of secondary metabolites, many of which are secreted and can be purified for use as pharmaceuticals, dyes, food additives and more. Many such compounds are complex, and include a variety of highly useful immunosuppressants, statins, antibiotics, chemotherapeutics and even vitamins (Barrios-González \& Miranda 2010, Survase et al. 2011, Kato \& Park 2012, Smith et al. 2014, Kück et al. 2014, Bachmann et al. 2014). 


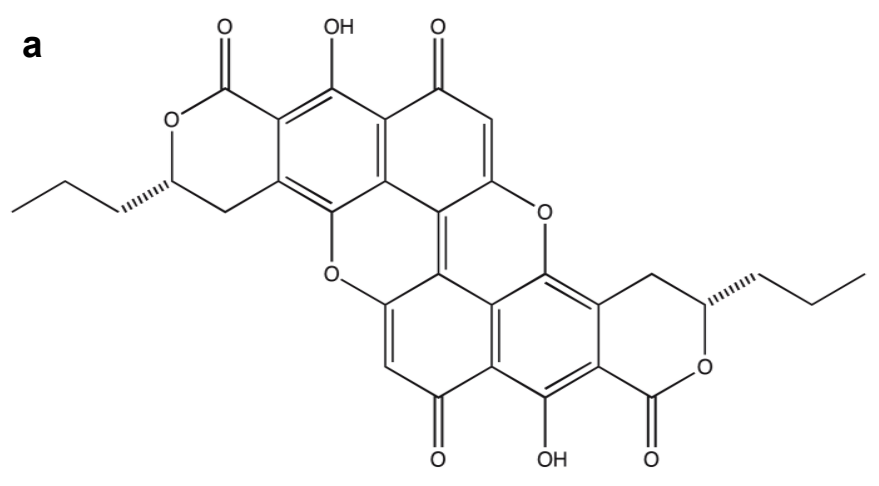

b

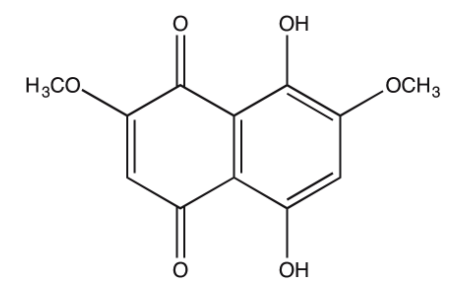

Fig. 1 - The structures of (a) xylindein secreted by $C$. aeruginosa and C. aeruginascens and (b) the red pigment secreted by $S$. cuboideum (Chemdraw, v15.0).

Pigment molecules produced by the wood-staining fungi Chlorociboria aeruginosa, $C$. aeruginascens, Scytalidium cuboideum, and S. ganodermophthorum are currently used in the decorative woods industry to increase the costs of low-value wood products through spalting, and were recently shown to have potential for use as textile dyes (Blanchette et al. 1992, Michaelsen et al. 1992, Robinson \& Laks 2010, Robinson et al. 2011a, Robinson et al. 2012, Tudor 2013, Robinson et al. 2014a, Weber et al. 2014). Chlorociboria aeruginascens and C. aeruginosa produce xylindein, the structure of which was first described in the mid-1960s by Blackburn et al. (1962), Edwards \& Kale et al. (1965), and Saikawa et al. (2000) (Fig 1a). Strategies for synthesizing conjugated hexacyclic heteroaromatic chalcogen-containing compounds are limited, and attempts to synthesize xylindein have thus far proven incomplete (Donner et al. 2012). Xylindein shows moderate solubility in water and high solubility in common organic solvents, with optimal solubility and stability over extended periods of time in DCM (Robinson et al. 2014a). Additionally, xylindein extracted from Chlorociboria aeruginosa and $C$. aeruginascens cultures shows long-term stability as a dry solid following solvent evaporation, and can be rehydrated for use in DCM, chloroform, chlorobenzene and similar. The high environmental stability of this molecule, as evidenced by still-colored intarsias and Tunbridge ware dating from the $15^{\text {th }}-19^{\text {th }}$ centuries, along with preliminary studies of xylindein under UV bombardment, suggest that the compound has significant potential for use as a commercial dye (Ellis 1976, Blanchette et al. 1992, Michaelsen et al. 1992, Schmidt 2006, Beck et al. 2014).

Scytalidium cuboideum produces, primarily, a pink-red pigment. The naphthoquinone derivative is produced by both bacterial and fungal species, and was first identified in Streptomyces by Gerber \& Wieclawek in 1966 (Fig 1b). The compound is also a degradation product of spinochrome M in sea urchins, and is reportedly produced by some Streptoverticillium species (Tresner et al. 1971, Schever 2012). Similar red pigments are secreted by the Monascus fungi, M. purpureus in particular, and are used as food colorings and additives in Japan and China (Feng et al. 2012). Production of the pigment by the fungus $S$. cuboideum was confirmed in 1995 from inoculated southern yellow pine (Pinus taeda L.) (Golinski et al. 1995). Under controlled conditions, $S$. cuboideum can infect and stain a wide variety of wood species, including both hardwoods and softwoods (Robinson et al. 2011a, Robinson et al. 2011b, Robinson et al. 2014c). The red pigment of S. cuboideum is one of the smallest molecular weight naturally-produced red pigments identified to date, and the extracted, dried pigment can be rehydrated in a wide variety of solvents (Golinski et al. 1995, Robinson et al. 2014a). 
Scytalidium ganodermophthorum produces a brown-yellow pigment. This fungus is best known as a causal agent of yellow rot of cultured Ganoderma lucidum (Kang et al. 2010, Kang \& Yun 2011). Like S. cuboideum and both of the Chlorociboria species, it has been shown to secrete its primary pigment into wood under controlled conditions (Robinson et al. 2014b). The structure of this yellow pigment has yet to be elucidated; however, like the red pigment from S. cuboideum, it is highly soluble in a wide range of solvents.

Current methods of growing the aforementioned fungi specifically for pigment production (in contrast to accelerated mycelial growth) involve the production of $2 \%$ malt agar plates amended with white-rotted wood dust (Robinson et al. 2012). Fungi are then inoculated onto the plates and allowed to grow until pigment has saturated the wood. The plates are then dried, ground up, and the pigment extracted using dichloromethane (DCM). Pigment extraction from stained wood from the forest floor can also be done in this manner. Inoculation of these fungi onto malt agar media, per the study above, does not produce significant extracellular pigment, even with months of incubation time.

While effective, this process is slow. For Chlorociboria fungi, the growth process above can take several months to full saturate a plate. In addition, the extraction requires a solvent such as DCM, chloroform, or pyridine (Robinson et al. 2014a). Amount of pigment generated is limited by the size of the plate and storage space, which creates another barrier to commercialization.

Growth of fungi in water-based systems, such as static liquid culture or shake culture, are established methods to increase growth and secondary metabolite production. However, each method has numerous factors, such as growth media, shake rate, temperature, etc., that effect what types of secondary metabolites are produced, and how quickly they are produced. Hence, the method must be idealized for each individual fungus based upon the desired results (Smania et al. 1997, Schreiner et al. 1998, Hamano et al. 2005, Ahn et al. 2006, among many others).

This work aimed to idealize the growing conditions for pigment production of $C$. aeruginascens, $C$. aeruginosa, $S$. cuboideum, and $S$. ganodermophthorum in liquid culture (without a agar or gelatin as a stabilizing agent). The ability to use spalting pigments directly from water-based systems, especially systems that can be easily scaled up to an industrial level, is the key to moving spalting from a traditional wood craft to an industrial-scale alternative to synthetic dyes.

\section{Materials \& Methods}

The following five fungal species and strains (two strains of $S$. cuboideum, one strain each of $C$. aeruginosa, $C$. aeruginascens and $S$. ganodermophthorum) were cultured for pigment collection: Chlorociboria aeruginosa (Oeder) Seaver (strain UAMH 11657, isolated from a hardwood log in Ontario, Canada), Chlorociboria aeruginascens (Nyl.) Kanouse (strain UAMH 7615, isolated in Lake District, UK), Scytalidium cuboideum (Sacc. \& Ellis) Sigler \& Kang (UAMH 4802, isolated from oak, location unknown; and UAMH 11517 isolated from Quercus sp. in Memphis, TN, USA) and Scytalidium ganodermophthorum Kang, Sigler, Lee \& Yun (UAMH 10320, isolated from an oak log in Gyeonggi Province, South Korea).

\section{Preliminary testing}

250mL Mason jars with plastic screw-cap lids were autoclaved for 30 minutes. Each jar contained enough $2 \%$ malt solution to just cover the bottom of the jar. After autoclaving, jars were allowed to cool overnight under a laminar flow hood with caps on. The next morning the jars were inoculated with a single point inoculation (approximately $2 \mathrm{~mm}^{3}$ in diameter) directly in the center of the jar, of one of the test fungi. Lids were screwed on tightly, then loosened $1 / 4$ turn. Parafilm was then wrapped around each lid. The jars were stored in a drawer under ambient temperature $\left(21^{0} \mathrm{C}\right)$ for one month, after which they were removed and visually analyzed.

\section{Shake culture and water analysis}

For each strain, $8.0 \mathrm{~g}$ of manually chopped fungal material (pieces of approximately $2 \mathrm{~mm}^{3}$ ) from a $10 \mathrm{~cm}$ plate of actively growing fungi ( $2 \%$ malt, $1.5 \%$ agar in deionized water) was inoculated into each of four $500 \mathrm{~mL}$ flasks containing $250 \mathrm{~mL}$ liquid media ( $2 \%$ malt in deionized water). Flasks were 
incubated at room temperature on a shaker at $110 \mathrm{rpm}$ for a period of 48 days, without media replenishment.

At four-day intervals, $3 \mathrm{~mL}$ of liquid media was collected from each flask, including controls. Media collected from cultures was centrifuged for $8 \mathrm{~min}$ at $8,000 \mathrm{rpm}$; supernatant containing the pigment was collected, and pelleted fungal material was discarded. Color readings were taken on a Konica Minolta CR-5 chroma meter with output in the CIE L*a*b* color space. Deionized water was used as a blank, and $\Delta \mathrm{E}$ values were calculated from $\mathrm{L}^{*}, \mathrm{a}^{*}$, and $\mathrm{b}^{*}$ values using liquid media alone as a comparison. $\Delta \mathrm{E}$ is defined by the following equation:

$$
\Delta \mathrm{E}^{*} \mathrm{ab}=\sqrt{\left(\Delta \mathrm{L}^{*}\right)^{2}+\left(\Delta \mathrm{a}^{*}\right)^{2}+\left(\Delta \mathrm{b}^{*}\right)^{2}}
$$

Centrifuged liquid culture samples were then analyzed using a Shimadzu HPLC instrument with an SPD-M20A photo diode array detector, and a phenyl-bonded column (Zorbax SB phenyl 4.6mm $\mathrm{x}$ $25 \mathrm{~cm}$ column, Agilent). Compounds present in the liquid media were eluted using a gradient of acetonitrile and $0.01 \% \mathrm{H}_{3} \mathrm{PO}_{4}$ (in water) over 10 minutes $(30: 70$ to $95: 5$, v/v), followed by a 5-minute column wash at $95 \%$ acetonitrile and a 5-minute column re-equilibration at $30 \%$ acetonitrile. The mobile phase flow rate was $1.5 \mathrm{~mL} / \mathrm{min}$, with a column oven and detector cell temperature of $40^{\circ} \mathrm{C}$. Peak areas for the pigments were calculated at $655 \mathrm{~nm}$ (blue-green xylindein), $506 \mathrm{~nm}$ (red pigment) and $449 \mathrm{~nm}$ (yellow pigment) as a result of UV-Visible spectroscopy findings with both the pigments in liquid culture media and the pigments extracted from liquid culture media in DCM.

As xylindein is poorly soluble in water, it was extracted directly from 48-day-old liquid cultures using DCM. Cultures were first blended in liquid media in a small electric blender (Oster Precise Blend 200, Model 6811). 100mL of DCM was poured directly into a culture flask containing blended media and fungal culture fragments, and the flask was either shaken at $150 \mathrm{rpm}$ or stirred using a PTFEcoated stir bar at $300 \mathrm{rpm}$. Media and DCM were poured together into a $500 \mathrm{~mL}$ separatory funnel. DCM containing pigment was collected from the funnel into a $250 \mathrm{~mL}$ flask, and liquid media with blended culture fragments was collected in a $500 \mathrm{~mL}$ flask for two additional extractions to remove any residual pigment. DCM containing pigment was combined from all three extractions, and was analyzed on the UV-Visible spectrophotometer.

\section{Solid phase extraction analysis}

Media collected from cultures was centrifuged for $8 \mathrm{~min}$ at $8,000 \mathrm{rpm}$; supernatant was collected and pelleted material was discarded. Pigment was separated from the majority of additional metabolites secreted into media using phenyl-bonded solid phase extraction (SPE) columns $(2 \mathrm{~g} / 12 \mathrm{~mL}$, Phenomenex, Torrance, CA, USA). Following column wetting, pigment in liquid media was loaded onto the top of the column. For both Chlorociboria species, interferents were washed from the column using $50 \%$ acetonitrile in water containing $0.01 \% \mathrm{H}_{3} \mathrm{PO}_{4}$, and xylindein was collected in either $100 \%$ acetonitrile or chloroform. For S. cuboideum, interferents were washed from the column using 30\% acetonitrile in water containing $0.01 \% \mathrm{H}_{3} \mathrm{PO}_{4}$, and the red pigment was collected in $100 \%$ acetonitrile. For $S$. ganodermophthorum, interferents were washed from the column using $35 \%$ acetonitrile in water containing $0.01 \% \mathrm{H}_{3} \mathrm{PO}_{4}$, and the yellow pigment was collected as a single band in either $100 \%$ acetonitrile or DCM. The identities of the cleaned pigments were verified by HPLC with UV-Vis spectroscopy.

\section{$U V$-Visible spectroscopy}

UV-Visible spectroscopy was performed using an HP Agilent 8453 Diode Array UV/VIS Spectrophotometer Model G1103A. Liquid culture samples were analyzed following centrifugation for $8 \mathrm{~min}$ at 8,000 rpm; supernatant was collected and pelleted material was discarded prior to analysis. Media alone (no inoculum) was used as a blank. Pigments extracted in DCM from 48-day-old liquid cultures were analyzed using DCM as a blank. SPE-cleaned samples were analyzed in the elution solvent directly, and the elution solvent alone was used as a blank. 


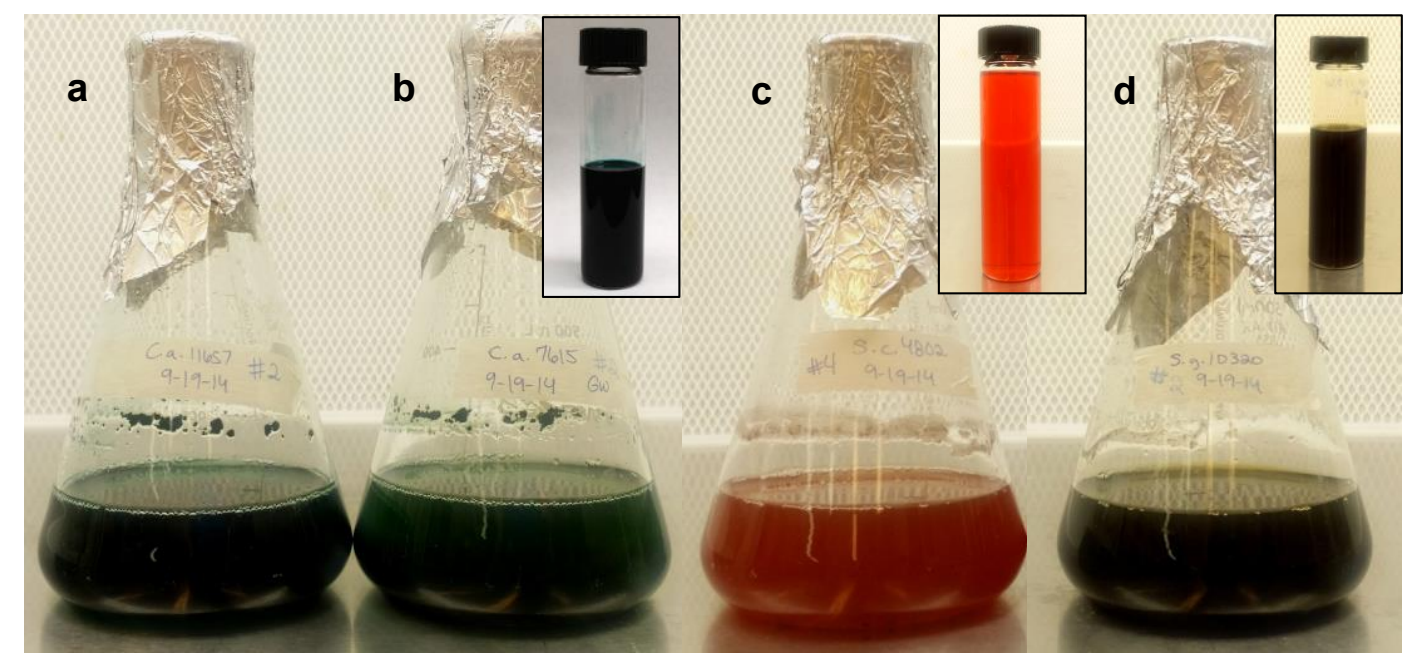

Fig. 2 - Liquid batch cultures at day 48. Xylindein in (a) C. aeruginosa 11657 culture and (b) $C$. aeruginascens 7615 culture. (c) The red pigment in $S$. cuboideum 11517 culture (appearance in culture is the same as $S$. cuboideum 4802, not shown), and (d) the yellow pigment in $S$. ganodermophthorum 10320 culture. Inserts show 8-dram glass vials containing the pigments extracted in DCM from liquid cultures and evaporated to the volumes shown.

\section{Mass spectrometry}

Mass spectrometry was performed using a Waters LCT Premier time-of-flight mass spectrometer coupled to a Shimadzu LC system at the Biomolecular Mass Spectrometry Core of the Environmental Health Sciences Core Center at Oregon State University. Media collected from cultures was centrifuged for $8 \mathrm{~min}$ at $8,000 \mathrm{rpm}$; supernatants containing the pigments were collected and analyzed, and pelleted fungal material was discarded. After 3 minutes at $30 \%$ acetonitrile, compounds were separated using a phenyl-bonded column (Zorbax SB phenyl $4.6 \mathrm{~mm}$ x $25 \mathrm{~cm}$ column, Agilent) and a gradient of acetonitrile and $0.01 \% \mathrm{H}_{3} \mathrm{PO}_{4}$ (in water) over 10 minutes (30:70 to 95:5, v/v), followed by a 5-minute column wash at $95 \%$ acetonitrile and a 5-minute column re-equilibration at $30 \%$ acetonitrile.

\section{Results}

While the Scytalidium species did produce extracellular pigment in static cultures, Chlorociboria species did not. Monitoring of the shake cultures showed visual extracellular pigmentation from all strains after one week (Fig 2a-b). Hence, trials continued only with shake cultures. As measured by both HPLC (peak area) and colorimeter $(\Delta \mathrm{E})$, media pigment levels climbed steadily through day 48 (without media replenishment) for $C$. aeruginosa 11657, and remained relatively unchanged after day 28 for C. aeruginascens 7615 (Fig 3). For both Chlorociboria species/strains, mycelial material tended to grow as discrete pellets, both in a shaken and stationary environment. When stationary, xylindein tended to aggregate in culture and to adhere to the glass of the culture flasks; this was alleviated by continually shaking the cultures at $110 \mathrm{rpm}$, although mats did eventually form even in shake culture. Blending of the solution before extraction did yield more pigment than simply extracting from the water-solubilized pigment, however sufficient pigment was suspended in the water that blending was not necessary to achieve visible pigment.

Pigmentation of the media for both $S$. cuboideum strains (initially a pink-red, and later a deep red) and for the $S$. ganodermophthorum strain (initially a light yellow, and later a deep yellow-red to brown with increasing pigment concentration) is observable in as few as $2-3$ days post-inoculation (Fig 2c-d). As measured by both HPLC (peak area) and colorimeter $(\Delta \mathrm{E})$, media pigment levels were observed at very similar levels for both S. cuboideum strains (Fig 4). Pigment levels initially declined from day three until observably different between day 16 and day 48, suggesting that pigment could be collected from $S$. cuboideum liquid cultures after just over two weeks incubation time. This somewhat unusual pattern of pigment production is reproducible under the conditions described, and can be observed after each new inoculation. 

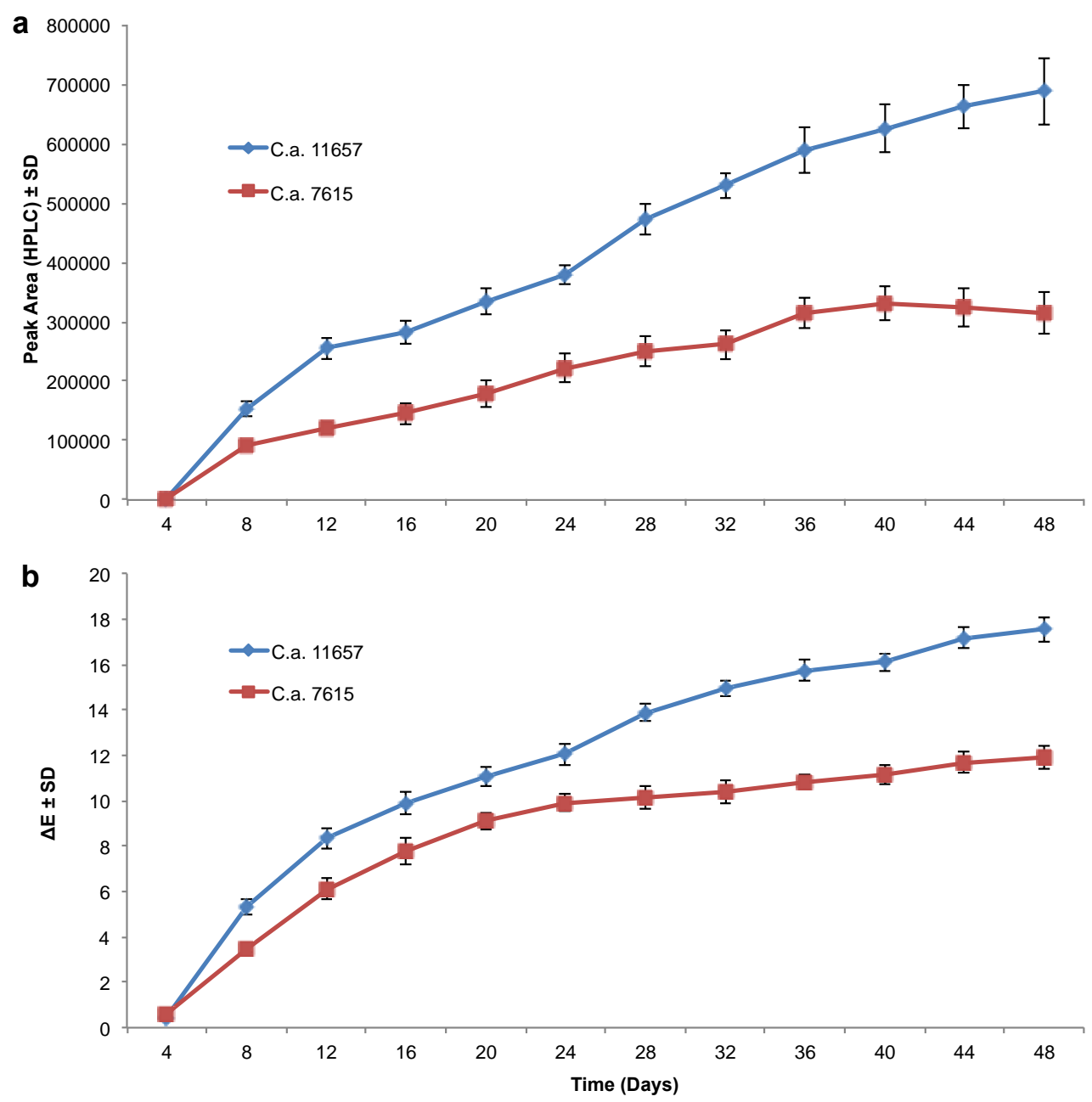

Fig. 3 - Production of xylindein from C. aeruginosa 11657 (C.a. 11657, blue lines with diamonds) and C. aeruginascens 7615 (C.a. 7615, red lines with squares) in liquid batch cultures as measured by (a) HPLC and (b) colorimeter. Error bars represent \pm 1 standard deviation.

S. ganodermophthorum produces yellow pigment at an enhanced rate until approximately day 8, when production begins to level off. Pigment levels in the media remained stable from approximately day 24 through day 48 (Fig 5). Unlike for S. cuboideum and the Chlorociboria species, the graph of $\Delta \mathrm{E}$ over time for $S$. ganodermophthorum liquid culture media was not entirely representative of HPLC peak area measurements (Fig $5 \mathrm{~b}$ ). $\Delta \mathrm{E}$ measurements would suggest that pigment production peaks on day 8 , after which it slowly declines until it begins to level out on day 24 , which does not closely match HPLC results. While $\Delta \mathrm{E}$ values have been shown to be useful when assessing pigment production patterns for liquid cultures in which a given color darkens over time (in the case of blue-green for $C$. aeruginosa and $C$. aeruginascens cultures or red for $S$. cuboideum cultures), when the culture media changes hue, $\Delta \mathrm{E}$ measurements lose their practicability.

This is because $\Delta \mathrm{E}$ measures color change in general; for example, a change from red to yellow may generate a similar $\Delta \mathrm{E}$ value as a change from yellow to darker yellow. In $S$. ganodermophthorum liquid cultures, the color of the media becomes steadily darker yellow over time, until it eventually appears a deep yellow-red to brown. Similarly, the pigment extracted directly from liquid media in DCM appears a bright yellow at lower concentrations and a deep yellow-red to brown at higher concentrations. While calculated $\Delta \mathrm{E}$ values are not useful in this case, $\mathrm{a}^{*}$ values alone, as generated by the colorimeter, did exhibit a similar pattern over time when compared to HPLC peak area measurements for $S$. ganodermophthorum liquid culture media (Fig 5c). This suggests that, for $S$. ganodermophthorum, $\mathrm{a}^{*}$ values, which represent red/green color transitions, are more representative of increasing yellow pigment concentration in liquid media than are $\Delta \mathrm{E}$ values. 

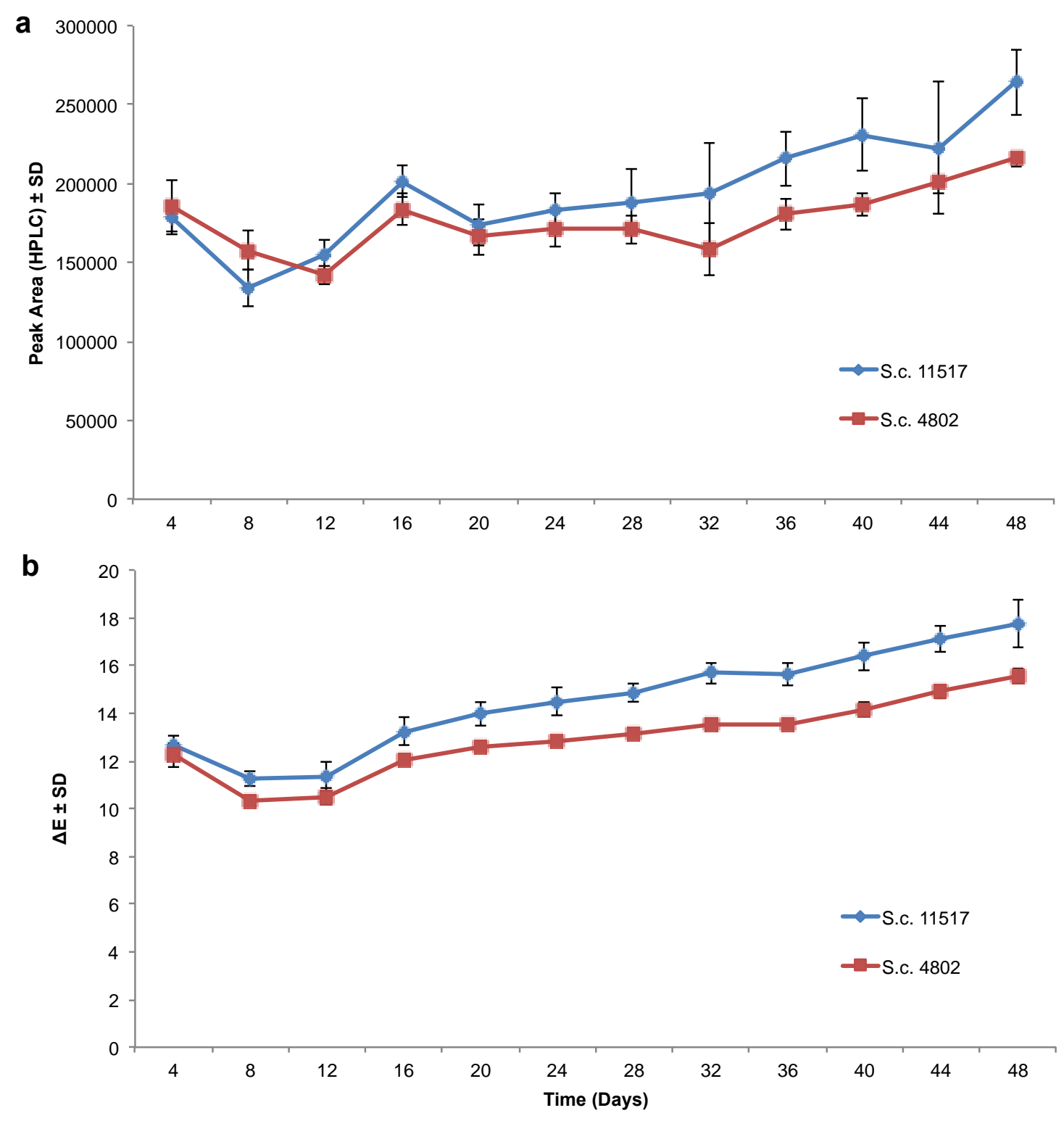

Fig. 4 - Production of the red pigment from S. cuboideum 11517 (S.c. 11517, blue lines with diamonds) and S. cuboideum 4802 (S.c. 4802, red lines with squares) in liquid batch cultures as measured by (a) HPLC and (b) colorimeter. Error bars represent \pm 1 standard deviation.

For both $S$. cuboideum and $S$. ganodermophthorum, mycelial material tended to grow as a single mat, generally resting toward the bottom of the culture flask (when stationary or shaking at lower rpms), or floating dispersed throughout the volume of the flask (at higher rpms). Pigment did not appear to aggregate for any of the three Scytalidium cultures, and did not adhere to the glass of the flask, in contrast to the xylindein pigment. The $S$. cuboideum mycelial mat appeared less heavily pigmented than the S. ganodermophthorum mat, which was colored a dark yellow by day 48 .

\section{Pigment extraction and analysis - confirming pigment identity}

Pigments, both in liquid culture media and extracted in DCM, were subjected to UV-Visible spectroscopy to identify appropriate wavelengths of detection for HPLC analysis. Spectral peaks from the analyses of liquid culture media (not shown) in all cases matched spectra obtained from pigments in DCM extracted from liquid culture media. Maximum absorbance for blue-green xylindein occurred at $655 \mathrm{~nm}$. Maximum absorbance for the red pigment occurred at $506 \mathrm{~nm}$. For the yellow pigment, maximum absorbance occurred at $449 \mathrm{~nm}$. 


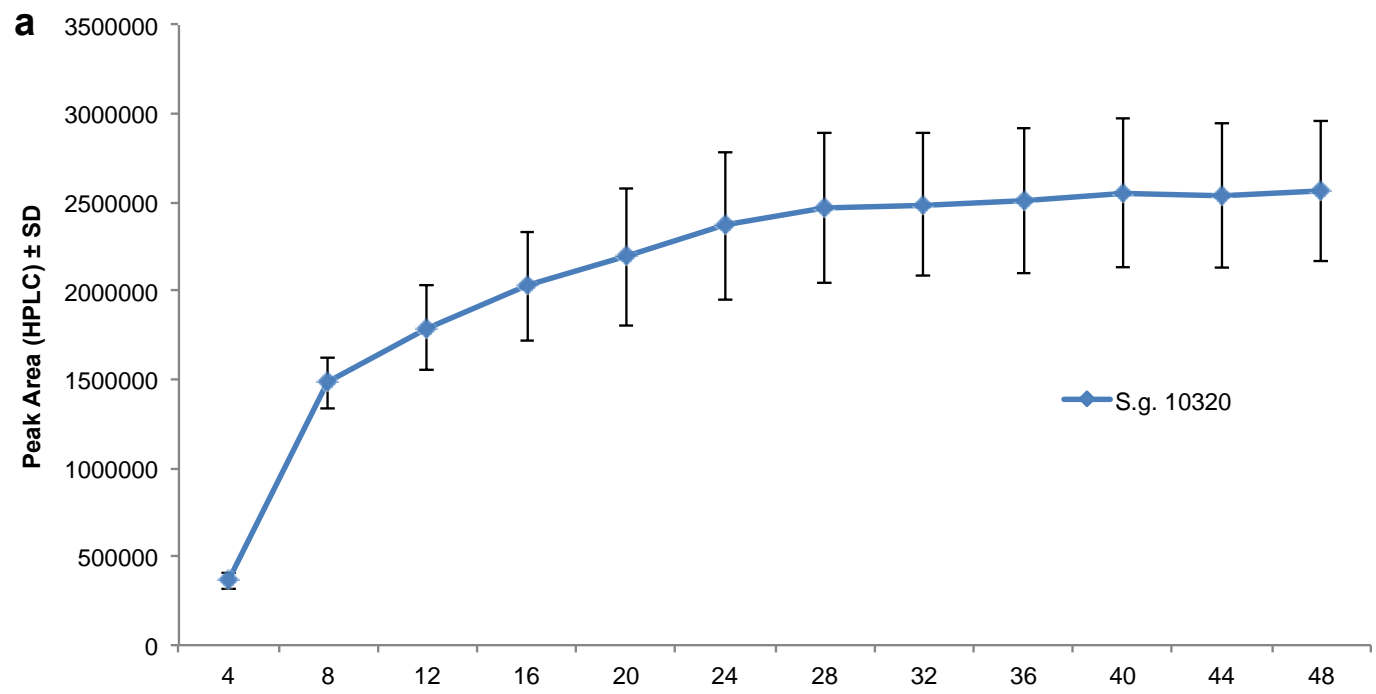

b
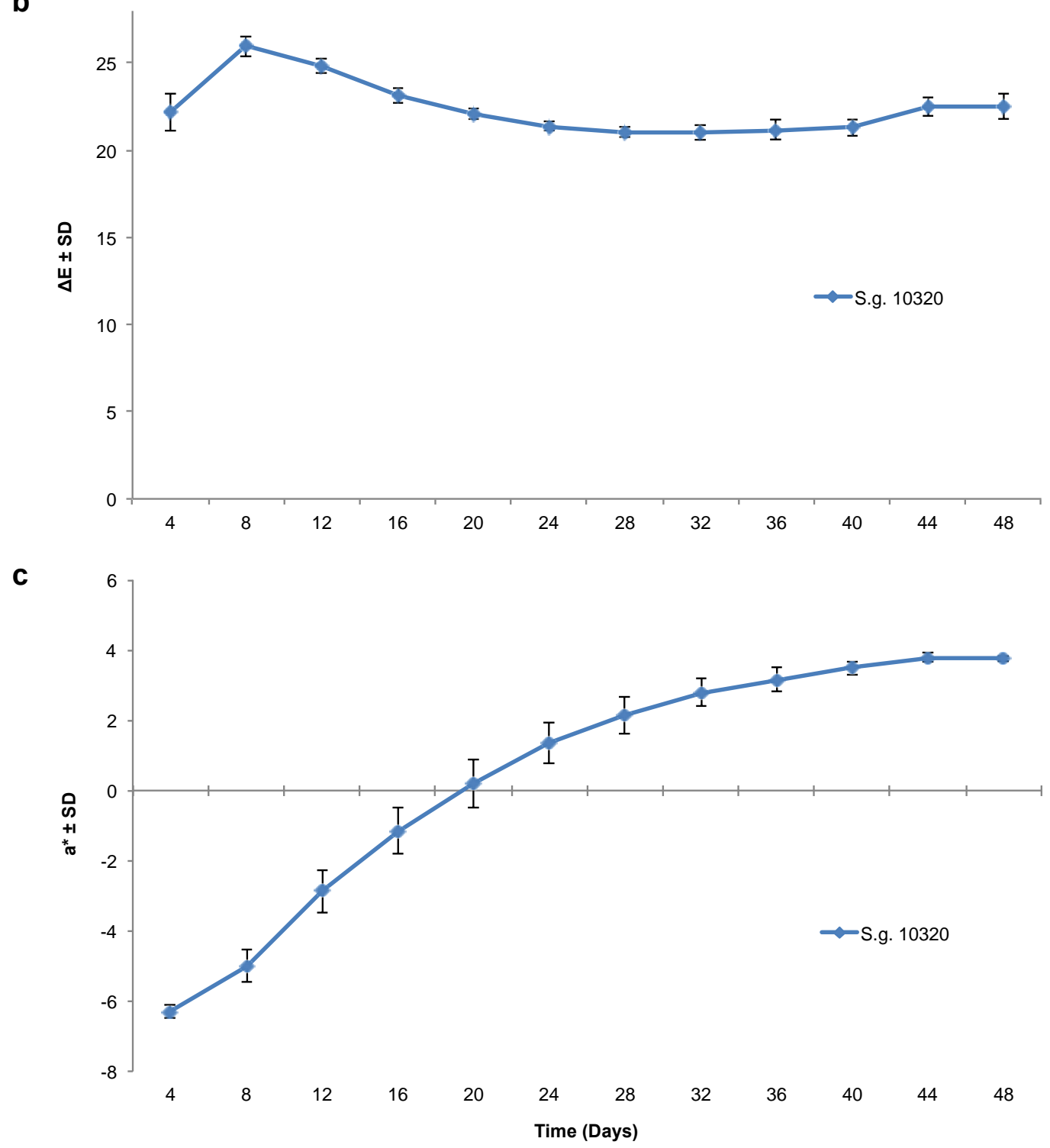

Fig. 5 - Production of the yellow pigment from $S$. ganodermophthorum in liquid batch cultures as measured by (a) HPLC and colorimeter; graphs of (b) $\triangle \mathrm{E}$ and (c) $\mathrm{a}^{*}$ over time are shown. Error bars represent \pm 1 standard deviation. 
Analysis of the pigments at these wavelengths during HPLC studies allowed for the cleanest possible peak integration during each time point. A UV-Vis spectrum for each pigment peak was also obtained using Shimadzu LCsolution HPLC software. For xylindein, $655 \mathrm{~nm}$ appeared as a valley between major peaks at $645 \mathrm{~nm}$ and $661 \mathrm{~nm}$. Additional peaks observed at $254 \mathrm{~nm}$ in the HPLC analysis of $C$. aeruginosa 11657 and $C$. aeruginascens 7615 liquid culture samples shared highly similar retention times and patterns between the two species; however, peak intensities (areas) for these additional media components, likely other secreted fungal metabolites, differed. Peaks observed at 254 $\mathrm{nm}$ and $506 \mathrm{~nm}$ in the HPLC analyses of S. cuboideum 11517 and S. cuboideum 4802 liquid culture samples appeared to be the same between the two strains.

High-resolution mass spectrometry of xylindein in C. aeruginosa 11657 and $C$. aeruginascens 7615 liquid cultures gave measured $m / z 569.1470$ and $569.1465[\mathrm{M}+\mathrm{H}]^{+}$, respectively, corresponding to the empirical formula $\mathrm{C}_{32} \mathrm{H}_{25} \mathrm{O}_{10}$ (calc'd 569.1148). Analysis of the red pigment in $S$. cuboideum 11517 and 4802 liquid cultures gave measured $\mathrm{m} / z 251.0559[\mathrm{M}+\mathrm{H}]^{+}$, corresponding to the empirical formula $\mathrm{C}_{12} \mathrm{H}_{10} \mathrm{O}_{6}$ (calc'd 251.0550). Analysis of the yellow pigment in S. ganodermophthorum 10320 liquid culture gave measured $\mathrm{m} / z 621.1961[\mathrm{M}+\mathrm{H}]^{+}$, corresponding to the empirical formula of $\mathrm{C}_{30} \mathrm{H}_{24} \mathrm{~N}_{10} \mathrm{O}_{6}$ (calc'd 621.1953).

For comparison and additional verification of the pigment peak in HPLC analyses of liquid culture samples, SPE-cleaned pigments were also analyzed via HPLC. The retention times of the major peaks from the cleaned pigments matched the retention times of the pigment peaks in liquid culture analysis. UV-Visible spectra were also obtained for the SPE-cleaned pigment peaks using the Shimadzu LCsolution HPLC software, and these spectra matched those obtained for the pigments from liquid cultures.

We identified the xylindein peak in liquid culture samples at a retention time of 12.75 min (approximately $95 \%$ acetonitrile in $0.01 \% \mathrm{H}_{3} \mathrm{PO}_{4}$ ) using the reported HPLC method. Notably, a yellow pigment was collected as a single band from SPE columns during the wash stage. We identified the red pigment peak at a retention time of $7.70 \mathrm{~min}$ (approximately $45 \%$ acetonitrile in $0.01 \% \mathrm{H}_{3} \mathrm{PO}_{4}$ ), and the yellow pigment peak at $10.30 \mathrm{~min}$ (approximately $65 \%$ acetonitrile in $0.01 \% \mathrm{H}_{3} \mathrm{PO}_{4}$ ).

\section{Discussion}

Analysis of the pigments did confirm presence of the target compounds. Maximum absorbance for blue-green xylindein occurred at $655 \mathrm{~nm}$, which agreed with previous studies of xylindein collected from intarsia artworks (Michaelsen et al. 1992). Maximum absorbance for the red pigment occurred at $506 \mathrm{~nm}$, which agreed with previous studies of the pigment collected from stained wood samples (Golinski et al. 1995). High-resolution mass spectrometry of xylindein gave measured $\mathrm{m} / \mathrm{z} 569.1470$ and $569.1465[\mathrm{M}+\mathrm{H}]^{+}$, respectively, corresponding to the empirical formula $\mathrm{C}_{32} \mathrm{H}_{25} \mathrm{O}_{10}$ (calc'd 569.1148), which is in agreement with the literature (Edwards \& Kale 1965, Saikawa et al. 2000). Analysis of $S$. cuboideum gave measured $\mathrm{m} / z 251.0559[\mathrm{M}+\mathrm{H}]^{+}$, corresponding to the empirical formula $\mathrm{C}_{12} \mathrm{H}_{10} \mathrm{O}_{6}$ (calc'd 251.0550), which is in agreement with the literature (Golinski et al. 1995).

Current spalting fungal culture techniques rely primarily on agar-based plates, with or without the inclusion of finely ground wood, and can take several months to reach maturity (Fenwick 1993, Robinson et al. 2012, Tudor 2013, Robinson et al. 2014a, Robinson et al. 2014b). The blue-green pigment, xylindein, is also commonly extracted from stained wood samples found in the natural environment; however, these samples are often difficult to find, and, given the relatively slow growth rates of Chlorociboria species in nature, naturally-pigmented wood samples are found only in limited quantities. Similarly, red-stained wood infected with $S$. cuboideum can be found in nature, but samples are rare. Outside of the laboratory, S. ganodermophthorum has thus far only been reported as a pathogen of reishi mushrooms, and collection of the yellow pigment has only occurred under controlled wood spalting or agar plate culture conditions (Kang et al. 2010, Kang \& Yun 2011, Robinson et al. 2014b, Robinson et al. 2014c).

Agar plate-based culture techniques and the collection of samples from nature are limiting in terms of producing process-scale quantities of fungal pigments over time. This preliminary work described simple, low-cost liquid batch culture conditions for the production of fungal pigments. 
Culturing spalting fungi in liquid media and extracting secreted pigments directly from this media has the added benefit of avoiding the extraction of any wood compounds, such as phenolics from lignin, that might preferentially solubilize in DCM along with the pigments, and necessitate additional cleaning steps.

While HPLC peak area data appear to be a more sensitive indicator of pigment levels in culture media, colorimeter data presented nearly the same patterns of pigment production, suggesting that a colorimeter (or a spectrophotometer) is a simple and convenient method for monitoring secreted pigment production under liquid culture conditions (Mapari et al. 2006, Pradeep et al. 2013). The results of this work show that $C$. aeruginosa strain 11657 produces greater quantities of blue-green xylindein over time under the described conditions than does $C$. aeruginascens strain 7615 . Additional strains of both $C$. aeruginascens and $C$. aeruginosa must be tested for levels of pigment production in liquid culture before conclusions can be drawn about differences in pigment production levels between the two species (rather than between the two strains in this study only). Interestingly, while HPLC data suggest that the additional fungal metabolites secreted into liquid media by $C$. aeruginosa 11657 and $C$. aeruginascens 7615 are highly similar, production patterns and levels over time for these additional media components differ somewhat between the two species. A yellow pigment, possibly a xylindein quinol (and potentially a breakdown product or precursor of the pigment), which has been observed by other groups, is also produced in liquid culture by Chlorociboria (Blackburn et al. 1962, Blackburn 1963, Edwards \& Kale 1965, Robinson et al. 2012). Notably, the compound peri-xanthenoxanthene, observed under laboratory conditions as a breakdown product of xylindein, is yellow, as is the tetraacetate (a derivative of xylindein quinol) detected by Kögl et al. (1925, 1930) following vigorous acetylation of the pigment (Kögl \& von Taeuffenbach 1925, Kögl \& Erxleben 1930, Blackburn et al. 1962, Blackburn 1963, Thomson 2012). As liquid cultures aged, we observed that the amount of this yellow pigment, collected prior to xylindein elution via SPE, decreased over time.

In our culture system, xylindein appeared to aggregate in liquid media at a limiting concentration, due to the low solubility of the pigment in aqueous solvents, although continual lowspeed (100-150 rpm) shaking greatly reduced this aggregation. We have found that blending the fungal material (in a commercial blender) prior to extraction with DCM greatly improves the efficiency of pigment extraction. In practice, pigment production in liquid cultures can be monitored using HPLC or spectrophotometry, and pigment can be extracted in DCM at the peak pigment production time point following blending of the culture material. However, blending of the mycelia in culture becomes problematic when continuous culturing and extraction are preferred. In the future, real-time extraction would be an ideal method of preventing pigment aggregation issues and would allow for efficient extraction of the pigment from living cultures on a continual basis.

While both Chlorociboria species/strains studied in this work grew as discrete pellets when inoculated from malt-agar plates into liquid culture (under both shaken and stationary conditions), other Chlorociboria strains isolated and currently under study in our laboratory have been observed to grow as mycelial mats at or near the top of the liquid culture media. Importantly, at higher volumes, the rheological properties of the liquid cultures, such as shear and viscosity, will directly influence shaker or mixer performance and bioreactor energy requirements. These and similar issues will vary depending on the characteristics of individual strains in liquid culture. These morphological differences translate into species- and even strain-specific variations in physiology and fungal growth kinetics in culture, and will need to be taken into consideration at larger scales. Studies on the growth of spalting fungi in liquid cultures are ongoing in our laboratory.

Unlike xylindein in liquid cultures, the red and yellow pigments produced by S. cuboideum and S. ganodermophthorum, respectively, do not appear to aggregate in aqueous solvents. However, both of these pigments exhibit superior solubility in non-aqueous solvents, including acetonitrile and DCM (Robinson et al. 2014a). Additionally, these pigments do not require blending of the liquid culture materials for their extraction from liquid culture media in DCM, although blending does result in a more efficient extraction. 


\section{Conclusions}

The results of this study demonstrate the successful culturing of the pigment-producing fungi, C. aeruginosa, $C$. aeruginascens, $S$. cuboideum and $S$. ganodermophthorum under low-cost liquid culture conditions. Pigment production can be effectively monitored over time using colorimetric analysis, although relative quantitation of pigments via HPLC analysis is a more sensitive measurement technique. This work presents an improved, effective and efficient method for the production of three industrially relevant fungal pigments under laboratory conditions. Ongoing work will focus on the absolute quantification of these pigments in liquid cultures, along with the growth kinetics of the four fungal species. Large-scale production and extraction of these pigments without interruption of culture systems, for example through the use of in-line microporous membrane liquid-liquid extraction methods, would further advance the cost-effectiveness and applicability of fungal pigments in the future.

\section{Acknowledgements}

We would like to acknowledge Dr. Jeff Morrell and Matthew Konkler of the Oregon State University Department of Wood Science and Engineering for their assistance with HPLC analyses. We would also like to acknowledge the assistance of Jeff Morre at the Biomolecular Mass Spectrometry Core of the Environmental Health Sciences Core Center at Oregon State University. The project described was supported in part by Award Number P30ES000210 from the National Institute of Environmental Health Sciences (NIEHS), National Institutes of Health (NIH). The content is solely the responsibility of the authors and does not necessarily represent the official views of the NIEHS or NIH.

\section{References}

Ahn J, Jung J, Hyung W, Haam S, Shin C. 2006 - Enhancement of Monascus pigment production by the culture of Monascus sp. J101 at low temperature. Biotechnology Progress 22, 338-340.

Bachmann BO, Van Lanen SG, Baltz RH. 2014 - Microbial genome mining for accelerated natural products discovery: is a renaissance in the making? J Ind Microbiol Biotechnol 41, 175-184.

Barrios-González J, Miranda RU. 2010 - Biotechnological production and applications of statins. Apple Microbiol Biotechnol 85, 869-883.

Beck H, Freitas S, Weber G, Robinson S, Morrell J. 2014 - Resistance of fungal derived pigments to ultraviolet light exposure. Proceedings of the $45^{\text {th }}$ Annual Meeting of the International Research Group on Wood Protection.

Blackburn GM. 1963 - Natures other greens. New Sci 19, 182-184.

Blackburn G. Neilson A, Todd A. 1962 - Structure of xylindein. Proc Chem Soc 327-328.

Blanchette RA, Wilmering AM, Baumeister M. 1992 - The use of green-stained wood caused by the fungus Chlorociboria in intarsia masterpieces from the $15^{\text {th }}$ century. Holzforschung 46, 225 232.

Donner CD, Cuzzupe AN, Falzon CL, Gill M. 2012 - Investigations towards the synthesis of xylindein, a blue-green pigment from the fungus Chlorociboria aeruginosa. Tetrahedron 68, 2799-2805.

Edwards R, Kale N. 1965 - The structure of xylindein. Tetrahedron 21, 2095-2107.

Ellis EA. 1976 - British Fungi, Part 2. Jarrold \& Sons, Norwich.

Feng Y, Shao Y, Chen F. 2012 - Monascus pigments. Appl Microbiol Biotechnol 96, 1421-1440.

Fenwick GA. 1993 - Chlorociboria aeruginascens in laboratory culture. Mycologist 7, 172-175.

Gerber NN, Wieclawek B. 1966 - The structures of two naphthoquinone pigments from an actinomycete. J Org Chem 31, 1496-1498.

Golinksi P, Krick TP, Blanchette RA, Mirocha CJ. 1995 - Chemical characterization of a red pigment (5, 8-dihydroxy-2, 7-dimethoxy-1, 4-naphthalenedione) produced by Arthrographis cuboidea in pink stained wood. Holzforschung 49, 407-410.

Hamano PS, Orozco SFB, Kilikian BV, 2005 - Concentration determination of extracellular and intracellular red pigments produced by Monascus sp. Brazillian Archives of Biology and Technology 48, 43-49. 
Kang H-J, Sigler L, Lee J, Gibas, CFC, Yun S-H, Lee Y-W. 2010 - Xylogone ganodermophthora sp. nov., an ascomycetous pathogen causing yellow rot on cultivated mushroom Ganoderma lucidum in Korea. Mycologia 102, 1167-1184.

Kang HJ, Yun SH. 2011 - Roles of ascospores and arthroconidia of Xylogone ganodermophthora in development of yellow rot in cultivated mushroom, Ganoderma lucidum. Plant Pathol J 27, 138-147.

Kato T, Park EY. 2012 - Riboflavin production by Ashbya gossypii. Biotechnol Lett 34, 611-618.

Kögl F, Erxleben H. 1930 - Untersuchungen über Pilzfarbstoffe. X. Über das Xylindein, den Farbstoff des grünfaulen Holzes (II). Justus Liebigs Ann Chem 484, 65-84.

Kögl F, von Taeuffenbach G. 1925 - Untersuchungen über Pilzfarbstoffe. IV. Über das Xylindein, den Farbstoff des grünfaulen Holzes (I). Justus Liebigs Ann Chem 445, 170-180.

Kück U, Bloemendal S, Teichert I. 2014 - Putting fungi to work: harvesting a cornucopia of drugs, toxins, and antibiotics. PLoS Pathog 10:e1003950.

Mapari SA, Meyer AS, Thrane U. 2006 - Colorimetric characterization for comparative analysis of fungal pigments and natural food colorants. J Agric Food Chem 54, 7027-7035.

Michaelsen H, Unger A, Fischer C-H. 1992 - Blaugrüne Färbung an Intarsienhölzern des 16. bis 8. Jahrhunderts: Wie kann sie identifiziert werden? Restauro 98, 17-25.

Pradeep FS, Begam MS, Palaniswamy M, Pradeep BV, Kumbf M. 2013 - Influence of culture media on growth and pigment production by Fusarium moniliforme KUMBF1201 isolated from paddy field soil. World Appl Sci J 22, 70-77.

Robinson SC, Hinsch E, Weber G, Freitas S. 2014a - Method of extraction and resolubilisation of pigments from Chlorociboria aeruginosa and Scytalidium cuboideum, two prolific spalting fungi. Color Technol 130, 221-225.

Robinson SC, Laks PE. 2010 - Wood species affects laboratory colonization rates of Chlorociboria sp. Int Biodeterior Biodegrad 64, 305-308.

Robinson SC, Tudor D, Cooper PA. 2011a - Wood preference of spalting fungi in urban hardwood species. Int Biodeterior Biodegrad 65, 1145-1149.

Robinson SC, Tudor D, Cooper PA. 2011b - Feasibility of using red pigment producing fungi to stain wood for decorative applications. Can J For Res 41, 1722-1728.

Robinson SC, Tudor D, Snider H, Cooper PA. 2012 - Stimulating growth and xylindein production of Chlorociboria aeruginascens in agar-based systems. AMB Express 2, 1-7.

Robinson SC, Tudor D, Zhang WR, Ng S, Cooper PA. 2014b - Ability of three yellow pigment producing fungi to colour wood under controlled conditions. Int Wood Prod J 5, 103-107.

Robinson SC, Weber G, Hinsch E, Vega Gutierrez SM, Pittis L, Freitas S. 2014c - Utilizing Extracted Fungal Pigments for Wood Spalting: A Comparison of Induced Fungal Pigmentation to Fungal Dyeing. Journal of Coatings, Article ID 759073.

Saikawa Y, Watanabe T, Hashimoto K, Nakata M. 2000 - Absolute configuration and tautomeric structure of xylindein, a blue-green pigment of Chlorociboria species. Phytochemistry 55, 237240.

Schever P. 2012 - Chemistry of marine natural products. Elsevier.

Schmidt O. 2006 - Wood and Tree Fungi: Biology, Damage, Protection, and Use. Springer.

Schreiner T, Hildebrandt U, Bothe H, Marner FJ. 1998 - Chemical and biological characterization of corticrocin, a yellow pigment formed by the ectomycorrhizal fungus Piloderma croceum. Zeitschrift fur Naturforschung C-A Journal of Biosciences 53, 4-8.

Smania EFA, Smania A Jr, Loguercia-Leite C, Gil ML. 1997 - Optimal parameters for cinnabarin synthesis by Pycnoporus sanguineus. Journal of Chemical Technology and Biotechnology 70, 57-59.

Smith KM, Gautschi JT, Freitag M. 2014 - Decoding the cryptic genomes of fungi: the promise of novel antibiotics. Future Microbiol 9, 265.

Survase SA, Kagliwal LD, Annapure US, Singhal RS. 2011 - Cyclosporin A-A review on fermentative production, downstream processing and pharmacological applications. Biotechnol Adv 29, 418-435. 
Thomson R. 2012 - Naturally occurring quinones. Elsevier.

Tresner HD, Hayes JA, Borders DB. 1971 - Production of a Naphthoquinone Pigment by a Species of Streptoverticillium and Its Accumulation by a Streptomycete. Appl Microbiol 21, 562.

Tudor D. 2013 - Fungal Pigment Formation in Wood Substrate. Thesis. University of Toronto.

Weber G, Chen H-L, Hinsch E, Freitas S, Robinson S. 2014 - Pigments extracted from the woodstaining fungi Chlorociboria aeruginosa, Scytalidium cuboideum, and S. ganodermophthorum show potential for use as textile dyes. Color Technol 130, 445-452. 